\title{
Isolated hemorrhage in the cerebellar vermis with vertigo and body lateropulsion to the contralesional side
}

\author{
Dong Hyun Lee, Se-Jin Lee
}

Department of Neurology, Yeungnam University of College of Medicine, Daegu, Korea

Received: June 22, 2019

Revised: August 20, 2019

Accepted: August 28, 2019

Corresponding author:

Se-Jin Lee

Department of Neurology,

Yeungnam University College of

Medicine, 170, Hyeonchung-ro

Nam-gu, Daegu 42415, Korea

Tel: +82-53-620-3683

Fax: +82-53-627-1688

E-mail:sejinmayo@ynu.ac.kr
There have been several reports of patients with isolated lesions of the cerebellar vermis presenting with clinical features similar to those of peripheral vestibulopathy. We report a case of small, isolated hematoma in the cerebellar vermis in a patient who presented with vertigo, ipsilesional nystagmus, and body lateropulsion to the contralesional side without the usual signs or symptoms of cerebellar dysfunction. Although they present with symptoms that mimic those of peripheral vestibulopathy, and brain computed tomography shows no abnormality, as there may be a small, isolated hematoma or infarction in the cerebellar vermis. Thus, brain magnetic resonance imaging should be performed in elderly patients with vascular risk factors.

Keywords: Cerebellar vermis; Hemorrhage; Nystagmus; Vertigo; Vestibulopathy

\section{Introduction}

Hemorrhage in the cerebellar vermis represents only $5 \%$ of all cases of cerebellar hemorrhage [1], which can easily rupture into the fourth ventricle through its roof and frequently extends into the pontine tegmentum. Prognosis of patients with hemorrhage in the cerebellar vermis is worse than that for overall cerebellar hemorrhages. Thus, cases of patients with a small hematoma in the cerebellar vermis, who present with clinical features of labyrinthine disorder without any other additional neurological deficits suggestive of a cerebellar lesion, are extremely rare, with only few reported in the literature [2-4]. We report a case of isolated hematoma in the vermis in a patient who presented with vertigo, ipsilesional nystagmus, and body lateropulsion to the contralesional side as the sole clinical manifestation.

\section{Case}

A 64-year-old woman with hypertension and hypercholesterol- emia presented to the emergency department with acute onset of severe non-positional vertigo accompanied by nausea, vomiting, and postural imbalance. Three days before presentation, she had experienced mild vertigo with nausea after sauna bathing, which improved with bed rest. At first, the patient had rotational vertigo aggravated by head motion and change of position. The following morning, her vertigo gradually worsened and persisted regardless of posture, until she eventually lost the ability to maintain even a sitting posture due to severe vertigo and disequilibrium. She had no other posterior circulation symptoms, such as diplopia, slurred speech, limb clumsiness or weakness, sensory loss, tinnitus, or hearing loss. A bedside neurological examination revealed a spontaneous right beating horizontal nystagmus with a torsional component in the primary position and upon gaze to the right or left. A bedside head impulse test was negative. There was no periodic alternating nystagmus. She had no skew deviation or head tilt and also showed no ophthalmoparesis, dysmetria, hypotonia, limb ataxia, or loss of proprioception. She fell to the left side when at-

Copyright(C) 2019 Yeungnam University College of Medicine

This is an Open Access article distributed under the terms of the Creative Commons Attribution Non-Commercial License (http://creativecommons.org/licenses/by-nc/4.0/) which permits unrestricted non-commercial use, distribution, and reproduction in any medium, provided the original work is properly cited. 
tempting to stand with her eyes open. All neurological findings suggested peripheral vestibulopathy, except the normal head impulse test finding. Brain computed tomography (CT) showed a subtle, small high-density region in the right cerebellum posterior to the fourth ventricle (Fig. 1). Brain magnetic resonance imaging (MRI) showed a small, acute stage hematoma selectively involving the nodulus of the right cerebellum located immediately posterior to the fourth ventricle with mild edema of the surrounding brain tissue (Fig. 2). Findings of brain magnetic resonance angiography (MRA) were unremarkable.

Severe vertigo, vomiting, and staggering gait continued for 1 week after admission. Thereafter, her symptoms gradually resolved, and she could walk without assistance after 2 weeks. Two months after presentation, she had no further dizziness or gait disturbance.

\section{Discussion}

There have been several reports of patients with isolated lesions of the cerebellar vermis presenting with clinical features similar to those of peripheral vestibulopathy without the usual signs or symptoms of cerebellar dysfunction, such as dysmetria, ataxia, asymmetrical pursuit and/or optokinetic nystagmus, and dysarthria [5-9]. The patterns of presenting signs and symptoms differ among patients depending on the location of the lesions. Isolated infarction of the nodulus can cause vertigo, nausea, vomiting, ipsilesional nystagmus, and body lateropulsion to the contralesional side and may, therefore, be clinically misdiagnosed as a labyrinthine disorder, which was previously reported as "pseudo-vestibular neuritis" syndrome $[5,6]$.

Cases of periodic alternating nystagmus, body lateropulsion, positional vertigo and nystagmus, pursuit eye movement disorders, or gait ataxia caused by lesions in various locations or different pathologies of the vermis have been reported [7-9]. Small cerebellar hemorrhage presenting with features similar to those of labyrinthine disorders has only been reported in a few cases, and not in the Korean literature [2-4]. In our case, $\mathrm{T}_{2}$-weighted gradient echo imaging showed a small hematoma in the caudal part of the lingula and the adjacent inferior-posterior white matter, immediately posterior to the fourth ventricle, in the right cerebellar vermis. This lesion caused acute vertigo, ipsilesional nystagmus, and body lateropulsion to the contralesional side. Isolated body laterpulsion can occur as a result of brain lesions at various locations, including the thalamus, cerebellum, medulla oblongata, and midbrain [5-7,10-14].

In the cerebellum, lesions of the nodulus, rostral vermis, tonsil, or cerebellar peduncle can develop isolated body lateropulsion [5-

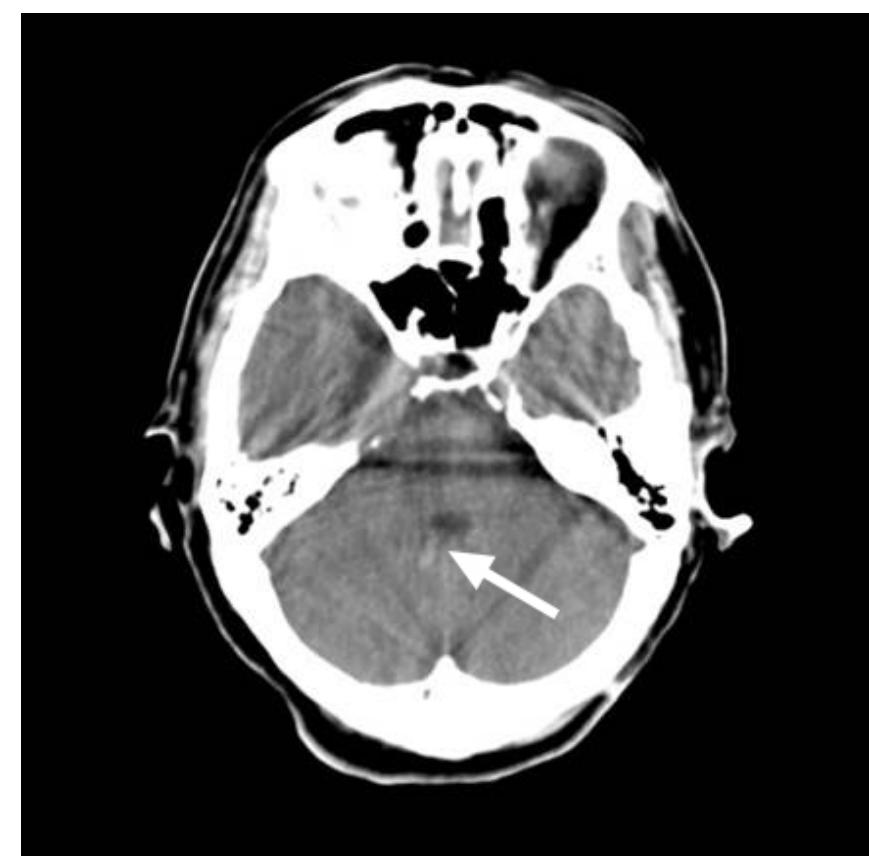

Fig. 1. Brain computed tomography image shows a high-density subtle, small region (arrow) in the vermis of the right cerebellum, posterior to the fourth ventricle.

7,9-11]. Body lateropulsion is contralesional in patients with lesions in the nodulus $[5,6]$, but ipsilesional in patients with lesions in the cerebellar peduncle, dorsal spinocerebellar tract, or rostral vermis $[7,10-14]$. However, our patient had lateropulsion to the contralesional side, ipsilesional nystagmus, and vertigo, which may be attributed to the location of the hematoma, which involved not only the lingula but also part of the nodulus and the white matter adjacent to the lingula. Spontaneous nystagmus and body lateropulsion in the nodulus lesion could be explained by disruption of nodular inhibition over the ipsilateral vestibular nuclei. The nodular Purkinje cell projects to the areas in the inferior vestibular nucleus, and a lesion in the nodulus may disinhibit the ipsilateral inferior vestibular nucleus, resulting in body lateropulsion to the contralateral side and ipsilateral spontaneous nystagmus [5].

The physician regarded the lesion as an artifact on the initial brain $\mathrm{CT}$, due to the very small hematoma size and subtle high-density of the lesion. Our patient presented with vertigo, ipsilesional nystagmus, and postural imbalance, with no additional signs or symptoms of cerebellar or brain stem dysfunction. However, brain MRI and MRA were performed to rule out a central vestibular lesion as the patient had vascular risk factors, such as hypertension, hypercholesterolemia, and old age. Caudal cerebellar infarction has been reported as the cause of isolated acute vertigo lasting for $>48$ hours in one-fourth of elderly patients who showed no abnormality on neurological examination, except for 

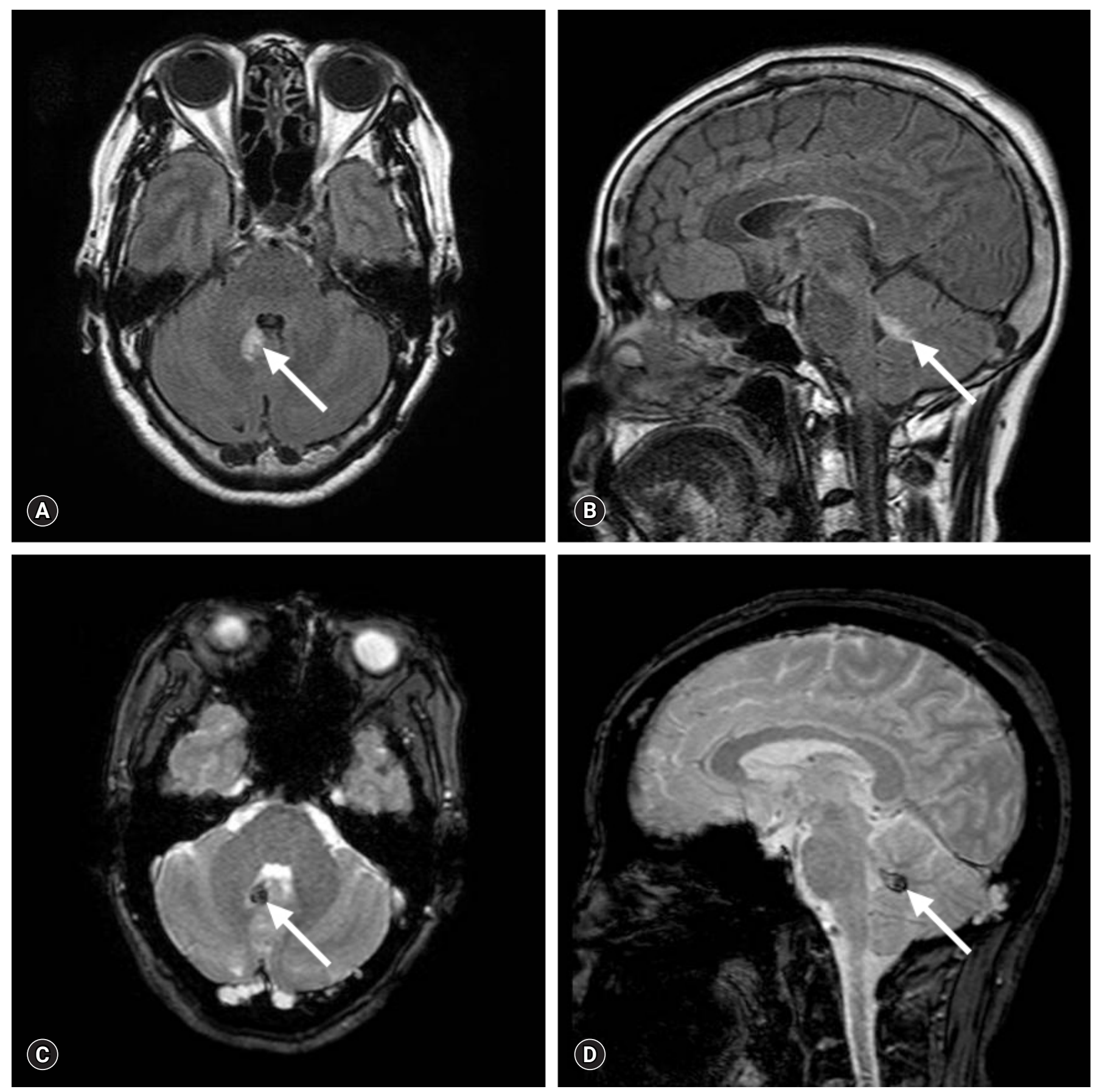

Fig. 2. Brain magnetic resonance imaging. Axial and sagittal fluid-attenuated inversion recovery imaging $(A, B)$ and $T_{2}$-weighted gradient echo imaging (C, D) show a small, isolated, acute stage hematoma (arrow) selectively involving the vermis of the right cerebellum located immediately posterior to the fourth ventricle, with mild edema of the surrounding brain tissue.

nystagmus. Therefore, brain MRI should be performed to differentiate cerebellar infarction from vestibular neuritis, especially in elderly patients with vascular risk factors [15]. Brain MRI clearly showed a small, acute stage hematoma in our case and is very helpful in patients with small hematomas restricted to the vermis, which may not be visible on brain CT. We concluded that the eti- ology of the hemorrhage in our case was spontaneous and hypertensive because the patient had no history of recent head trauma and vascular anomaly on MRI and MRA, and furthermore, the coagulation system was normal.

In summary, we report the case of a patient with small, isolated hemorrhage in the cerebellar vermis who presented with acute 
vertigo and body lateropulsion to the contralesional side. We believe that elderly patients, especially those with vascular risk factors, need to be examined carefully and brain MRI should be performed to rule out the possibility of a cerebellar lesion, even though patients present with signs and symptoms suggestive of acute peripheral vestibulopathy. MRI allows for the accurate detection of even small, isolated hematomas or infarctions located in the cerebellar vermis.

\section{Conflicts of interest}

No potential conflicts of interest relevant to this article was reported.

\section{ORCID}

Dong Hyun Lee, https: / /orcid.org/0000-0002-4434-8901

Se-Jin Lee, https:/ / orcid.org/0000-0001-7438-691X

\section{References}

1. Kase CS, Mohr JP, Caplan LR. Intracerebral hemorrhage. In: Mohr JP, Choi DW, Grotta JC, Weir B, Wolf PA, editors. Stroke: pathophysiology, diagnosis, and management. 4th ed. Philadelphia: Churchill Livingstone; 2004. p. 327-76.

2. Kattah JC, Kolsky MP, Luessenhop AJ. Positional vertigo and the cerebellar vermis. Neurology 1984;34:527-9.

3. Huang CY, Yu YL. Small cerebellar strokes may mimic labyrinthine lesions. J Neurol Neurosurg Psychiatry 1985;48:263-5.

4. Johkura K. Central paroxysmal positional vertigo: isolated dizzi- ness caused by small cerebellar hemorrhage. Stroke 2007;38: e26-7.

5. Lee H, Yi HA, Cho YW, Sohn CH, Whitman GT, Ying S, et al. Nodulus infarction mimicking acute peripheral vestibulopathy. Neurology 2003;60:1700-2.

6. Lee $\mathrm{H}$, Cho YW. A case of isolated nodulus infarction presenting as a vestibular neuritis. J Neurol Sci 2004;221:117-9.

7. Lee H. Isolated body lateropulsion caused by a lesion of the rostral vermis. J Neurol Sci 2006;249:172-4.

8. Oh YM, Choi KD, Oh SY, Kim JS. Periodic alternating nystagmus with circumscribed nodular lesion. Neurology 2006;67:399.

9. Muley SA, Bushara KO. Isolated gait ataxia due to cerebellar vermis infarct. Arch Neurol 2004;61:1461.

10. Shan DE, Wang V, Chen JT. Isolated lateropulsion of the trunk in cerebellar infarct. Clin Neurol Neurosurg 1995;97:195-8.

11. Bertholon P, Michel D, Convers P, Antoine JC, Barral FG. Isolated body lateropulsion caused by a lesion of the cerebellar peduncles. J Neurol Neurosurg Psychiatry 1996;60:356-7.

12. Lee H, Sohn CH. Axial lateropulsion as a sole manifestation of lateral medullary infarction: a clinical variant related to rostral-dorsolateral lesion. Neurol Res 2002;24:773-4.

13. Kim SH, Cho J, Cho JH, Han SW, Kim SM, Park SC, et al. Isolated lateropulsion by a lesion of the dorsal spinocerebellar tract. Cerebrovasc Dis 2004; 18:344-5.

14. Thömke F, Marx JJ, Iannetti GD, Cruccu G, Fitzek S, Urban PP, et al. A topodiagnostic investigation on body lateropulsion in medullary infarcts. Neurology 2005;64:716-8.

15. Norrving B, Magnusson M, Holtås S. Isolated acute vertigo in the elderly; vestibular or vascular disease? Acta Neurol Scand 1995;91:43-8. 